\title{
An Uncommon Intramedullary Tumor: Primary Spinal Cord Melanoma
}

\author{
Nuri Eralp Çetinalp, Ali Erdem Yildirim, Denizhan Divanlioglu, Deniz Belen \\ Department of Neurosurgery, Ankara Numune Education and Research Hospital, Ankara, Turkey
}

\begin{abstract}
A 47-year-old woman was admitted with complaints of progressive weakness in the lower extremities and pain in the back and left leg. Thoracic magnetic resonance imaging (MRI) revealed a spinal intramedullary tumor between the T9 and L1 levels, which were iso- to hypointense on T2 and slightly hyperintense on T1-weighted images. The tumor was resected as total, and the diagnosis was malignant melanoma confirmed with histopathology. Neurological findings improved at the postoperative period and no residual or recurrence was noted on postoperative MRI at the 9-month follow-up. Primary melanoma of the spinal cord, particularly intramedullar localization, is seldomly reported in the literature. We report a primary malignant melanoma of the spinal cord and emphasize the diagnostic and prognostic challenges.
\end{abstract}

Keywords: Spinal cord neoplasms; Intramedullary; Malignant melanoma

\section{Introduction}

Primary malignant melanoma of the central nervous system (CNS) accounts for only1\% of all melanoma cases, although they are the third most common cause of CNS metastases [1]. Primary melanoma of the spinal cord is a rare entity. So far, only 40 cases were reported since the first case reported by Hirschberg in 1906. Therefore, the exact incidence is still unclear. We report a case of primary malignant melanoma of the spinal cord and emphasize the diagnostic and prognostic challenges in the light of the foregoing literature.

\section{Case Report}

A 47-year-old woman was admitted to our clinic with complaints of progressive worsening of severe back pain radiating to the left leg for over a course of six months. Weakness in the lower extremities, which began a few weeks ago, was another prominent complaint, and no remarkable finding was noted in her past medical history. Neurological examination revealed moderate paraparesis and numbness predominantly on the left lower extremity in which motor strength was assessed to be $3 / 5$ in all the muscle groups, whereas the right lower extremity had 4/5 strength. Magnetic resonance imaging (MRI) of the thoracic spine showed an intramedullary spinal cord tumor between the T9 and L1 levels. The lesion was iso- to hypointense on T2 and slightly hyperintense on T1-weighted images. Diffuse contrast enhancement was also prominent on T1-weighted images after gadolinium injection (Fig. 1). The MRI images also showed a long segment of syrinx with multiple internal septa above the tumor from the T2 to T9 levels, and another syrinx cavity

Received Mar 30, 2013; Revised May 5, 2013; Accepted Sep 1, 2013

Corresponding author: Nuri Eralp Çetinalp

Department of Neurosurgery, Ankara Numune Education and Research Hospital,

Talatpasa Blvd. No:5 Altındag, Ankara, Turkey

Tel: +90-532-780-3775, Fax: +90-312-311-4340, E-mail: eralpmd@yahoo.com 
below the tumor at the levels of L1-L2. The preliminary diagnosis was ependymoma based on the MRI findings, where there were a well-circumscribed mass and a nonenhancing tumor with the associated rostral and caudal cyst.

During the operation, laminectomies were performed from T9 to L1 levels. A dark gray vascular tumor was observed immediately after a dural incision (Fig. 2). This pigmented tumor showed clear pial invasion under the operative microscope. The tumor was hardly dissected from the spinal cord, particularly at the caudal pole. The tumor was excised grossly as total. Postoperative course was uneventful. Although she had no recovery in motor strength in the early postoperative period, motor findings and pain radiating from the back to the left lower extremity markedly improved one month after the operation. No residual or recurrence was noted on postoperative MRI at 9 months of follow-up.

Histopathological sections demonstrated a highly cellular lesion composed of clusters of atypical cells with prominent nucleoli and marked eosinophilic cytoplasm. İmmunohistochemical staining revealed positive immunoreactivity for S100 protein and human melanoma black-45 (Fig. 3).

The patient underwent a thorough systemic survey after confirmation of the diagnosis, including tumor markers, ophthalmological and dermatological examinations. Any other foci of melanoma could not be found and primary malignant melanoma was confirmed. The patient did not receive any radiotherapy or chemotherapy and was able to walk without support at the sixth-month follow-up.

\section{Discussion}

Although most melanomas arise from the skin primarily, noncutaneous sites, such as gastrointestinal system

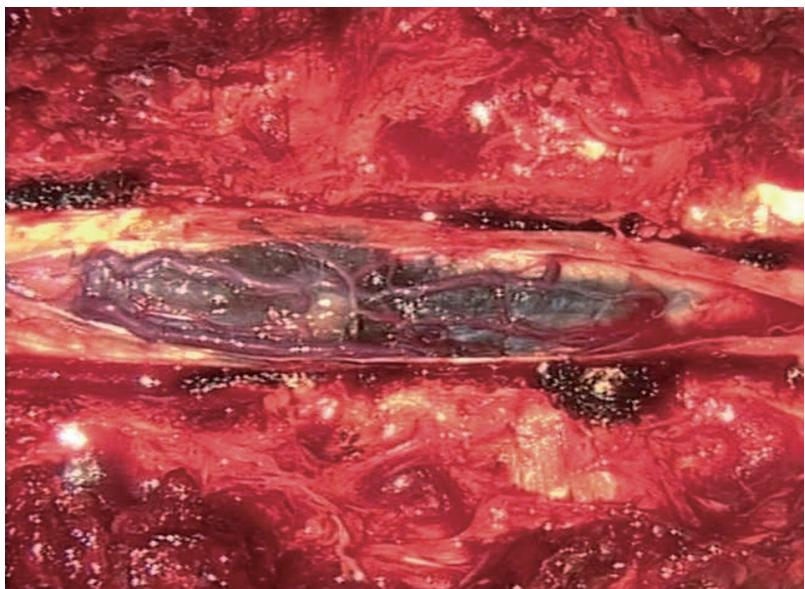

Fig. 2. Operation microscope view of the surgical area. Dark gray pigmented intramedullary lesion is exposed after dural incision.
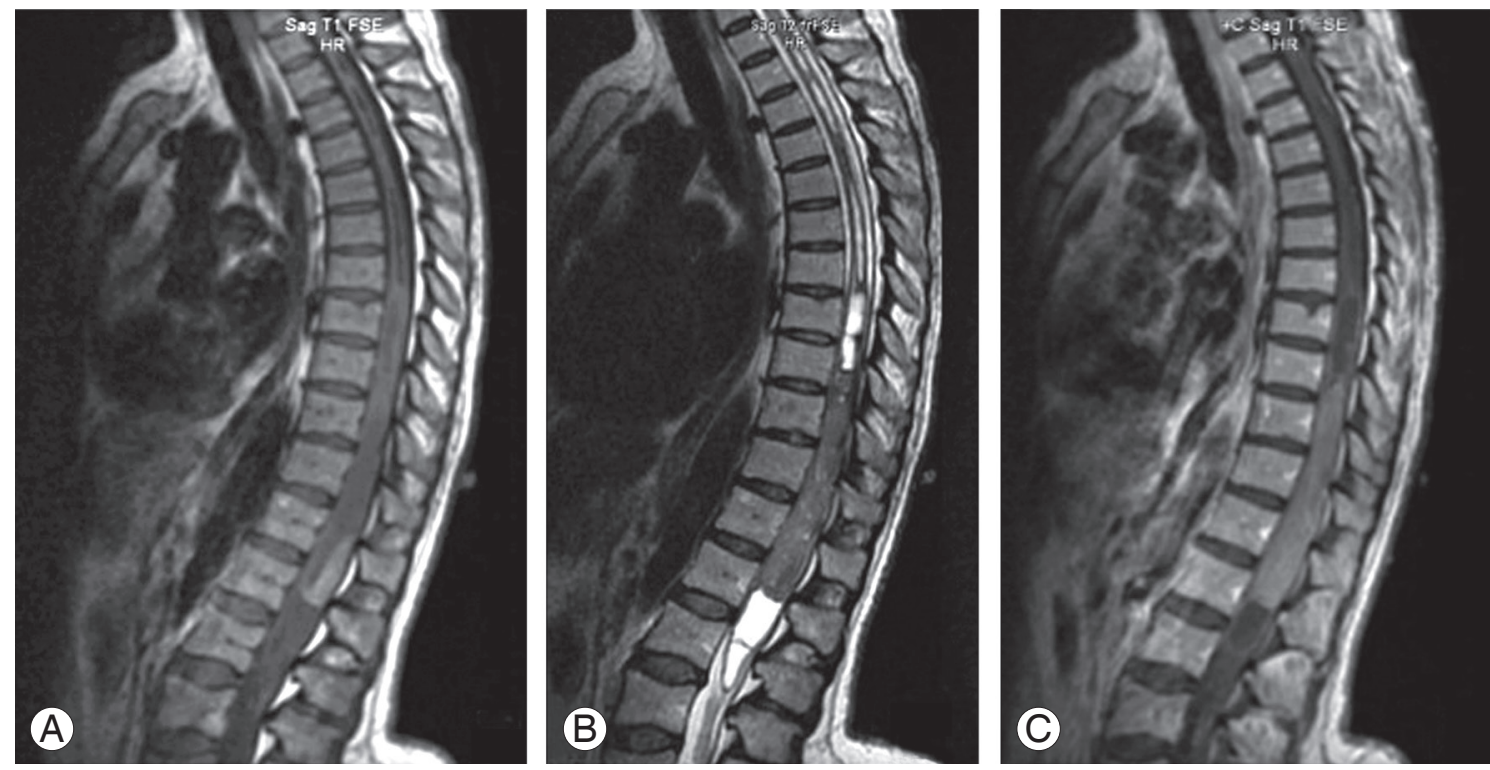

Fig. 1. Sagittal magnetic resonance imaging of the thoracic spine demonstrating an intramedullary spinal cord tumor between T9 and L1 levels. (A) The lesion is slightly hyperintense on T1-weighted images (B) iso- to hypointense on T2 weighted images with caudal and rostral cysts with multiple internal septa at the levels of T2 to T9 and L1 to L2. (C) Gadolinium enhanced T1-weighted images show mild homogeneous enhancement of the lesion. 
and ocular locations, were also reported [2]. Among all, spinal cord melanomas are rare tumors and are believed to originate from leptomeningeal melanoblasts, which are derived from the neural crest during early embryonic development [3].

Primary spinal melanoma is most frequently encountered at the thoracic region, as reported by Fuld et al. [2], and more than half of the cases were observed in this area.

MRI is still the best imaging modality for a spinal cord tumor, depending on signal variations at selected sequences and contrast enhancement patterns; however, it is still insufficient in differential diagnosis. Spinal cord melanomas were reported to be slightly hyperintense on T1, and iso- to hypointense on T2 weighted images. These tumors show homogeneous mild enhancement after intravenous gadolinium injection. The paramagnetic properties of melanin or hemorrhagic elements in the tumor were reported to be responsible for these signal intensity features of melanocytic tumors [4]. Thus, a malignant melanoma may be considered as nonspecific in terms of imaging features, and a precise diagnosis is difficult to assert before a histopathological verification. Yet, a differentiation between primary and metastatic spinal cord melanoma origin is another challenge because the primary melanoma of the CNS has a long-term survival rate than the metastatic type.

Life expectancy is usually less than a year in metastatic melanoma. On the other hand, the mean survey for primary CNS melanomas are reported to be approximately 6 years [5]. The primary melanoma of the spinal cord is diagnosed according to Hayward's criteria [6]: Absence of malignant melanoma outside the CNS and histopathological confirmation.

Surgical resection seems to be the best choice for primary spinal cord melanomas. Postoperative radiotherapy or chemotherapy was recommended by several authors; however, clear evidence with regard to their efficacy is
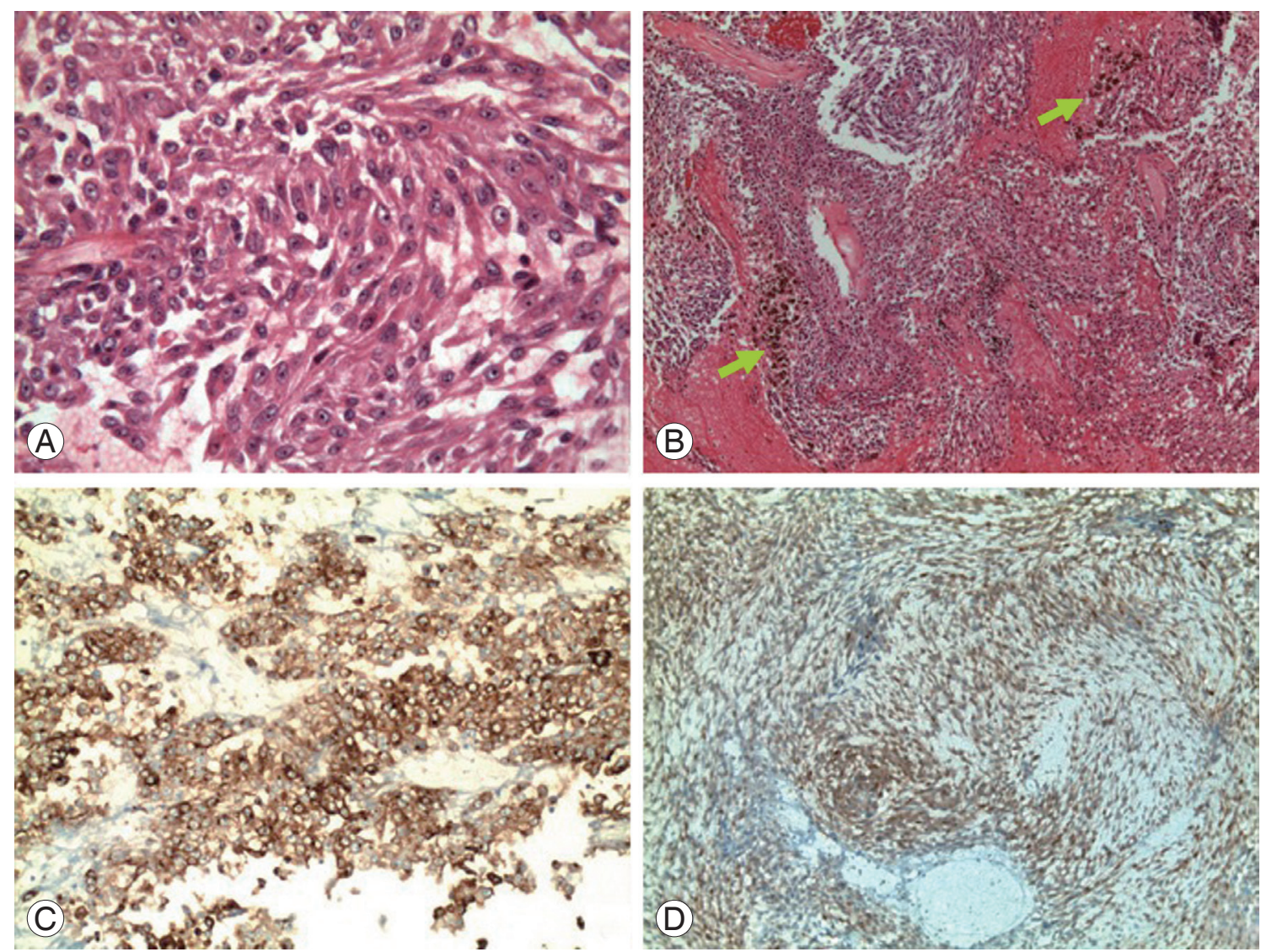

Fig. 3. Histopathological studies. (A) Photomicrograph shows highly cellular lesion composed of clusters of atypical cells with prominent nucleoli and marked eosinophilic cytoplasm (H\&E, $\times 400)$. (B) Green arrows indicate dark brown pigmentation indicating the presence of melanin. (C) Positive immunoreactivity for HBM-45 (×200) and (D) S-100 protein (x200). 
still unproven [7]. However, long-term survival after partial removal of a primary spinal melanoma followed by radiotherapy, chemotherapy and intratechal interferonbeta injection was also reported to be beneficial as well [8]. Kim et al. [1] reported a mean survey of 29 months ranging from 3 to 156 . Histopathological features were responsible for these marked variations in the survival rates regarding melanomas. Uveal melanomas were classified by Callender et al. into three groups according to the shape and differentiation status of the cells: Spindle A cells, spindle B cells and epithelioid cells (pleomorphic) [1]. McLean et al. [9] reported that pure spindle cell tumors have the best prognosis regardless of type, and the worst prognosis was seen in epithelioid cell tumors. CNS melanocytic neoplasms have a tendency to show the same properties, and this may be the reason for the various reports regarding different survival rates [10]. A prognostic study depending on the histopathological type could not be established because these tumors are seldom. A follow up, including MRI studies, is essential due to the unpredictable clinical course of the disease.

Primary spinal cord melanomas are seldomly reported and the crucial point about these malignancies is differentiation from a metastatic cutaneous melanoma due to its relatively better prognosis. In this report, we also want to stress the difference of the signal pattern of these tumors on MRI, which is iso- to hypointense in both T1 and T2 images, unlike most of the intramedullary tumors. We believe that the clinician must be aware of the possibility of a primary malignant melanoma when the MRI images depict such a spinal cord tumor with paramagnetic properties.

\section{Conflict of Interest}

No potential conflict of interest relevant to this article was reported.

\section{References}

1. Kim MS, Yoon DH, Shin DA. Primary spinal cord melanoma. J Korean Neurosurg Soc 2010;48:157-61.

2. Fuld AD, Speck ME, Harris BT, et al. Primary melanoma of the spinal cord: a case report, molecular footprint, and review of the literature. J Clin Oncol 2011;29:e499-502.

3. Pappenheim E, Bhattacharji SK. Primary melanoma of the central nervous system. Clinical-pathological report of a case, with survey and discussion of the literature. Arch Neurol 1962;7:101-13.

4. Farrokh D, Fransen P, Faverly D. MR findings of a primary intramedullary malignant melanoma: case report and literature review. AJNR Am J Neuroradiol 2001;22:1864-6.

5. Larson TC 3rd, Houser OW, Onofrio BM, Piepgras DG. Primary spinal melanoma. J Neurosurg 1987;66: 47-9.

6. Hayward RD. Malignant melanoma and the central nervous system. A guide for classification based on the clinical findings. J Neurol Neurosurg Psychiatry 1976;39:526-30.

7. Patchell RA, Tibbs PA, Regine WF, et al. Direct decompressive surgical resection in the treatment of spinal cord compression caused by metastatic cancer: a randomised trial. Lancet 2005;366:643-8.

8. Nishihara M, Sasayama T, Kondoh T, Tanaka K, Kohmura E, Kudo H. Long-term survival after surgical resection of primary spinal malignant melanoma. Neurol Med Chir (Tokyo) 2009;49:546-8.

9. McLean IW, Saraiva VS, Burnier MN Jr. Pathological and prognostic features of uveal melanomas. Can J Ophthalmol 2004;39:343-50.

10. Brat DJ, Giannini C, Scheithauer BW, Burger PC. Primary melanocytic neoplasms of the central nervous systems. Am J Surg Pathol 1999;23:745-54. 